PROCEEDINGS OF THE AMERICAN MATHEMATICAL SOCIETY

Volume 124, Number 1, January 1996

\title{
SYMPLECTIC LEAVES AND DEFORMATION QUANTIZATION
}

\author{
ALBERT J. L. SHEU
}

(Communicated by Palle E. T. Jorgensen)

\begin{abstract}
In this paper, we show that for any classical simple compact Poisson Lie group $K$, there is no quantization of $K$ using the quantum group $K_{q}$,
\end{abstract} which is both group-preserving and symplectic leaf-preserving.

\section{INTRODUCTION}

The subject of quantum groups $K_{q}$ associated to classical simple compact matrix groups $K$ has been extensively studied by many people from different viewpoints [D, Re-T-F, Le-So, So-V, Wo2]. It is fair to say that a major interest in quantum groups arose from the use of them to quantize the underlying group structure and Poisson structure [Ri1, Ri3, Sh1, Sh2]. Our paper answers an interesting question related to this quantization aspect.

To state our result in a very general form, we shall call any unital linear map sending regular functions on $K$ to elements in $C\left(K_{q}\right)$ a quantization. Since the group multiplication at quantum level is reflected by the coalgebra structure on $C\left(K_{q}\right)$, we say that a quantization is group-preserving if it is a coalgebra homomorphism. On the other hand, the remarkable work of Soibelman [So] (cf. also [Le-So, Sh4]) showed that the structure of the function algebra $C\left(K_{q}\right)$ of $K_{q}$ is closely related to the underlying symplectic foliation, the main geometric object given by a Poisson structure [We]. From their result, one gets the notion of quantum symplectic leaves and the notion of leaf-preserving quantization. In view of the known examples of group-preserving or leaf-preserving deformation quantizations of $S U(2)$ [Sh2, Sh1], it is a natural question to ask whether there is a quantization which is both group-preserving and leaf-preserving at the same time [Ri2]. In this paper, by combining Soibelman's result and the negative answer for $S U(2)$ obtained in [Sh3], we get a negative answer to this question for all $K$.

\section{QuANTUM LEAVES OF $K_{q}$}

In this section, we recall the notion of quantum leaves arising from Soibelman's classification of irreducible ${ }^{*}$-representations of the function algebras $C\left(K_{q}\right)^{\infty}$ of simple compact matrix quantum groups $K_{q}$, and refer readers to the papers [So, Le-So] for details.

Received by the editors June 21, 1994.

1991 Mathematics Subject Classification. Primary 46L87, 81R50.

Partially supported by NSF-Grant DMS-9303231.

(C)1996 American Mathematical Society 
Let $K$ be a maximal compact subgroup of a simple complex Lie group $G$, and let $\mathfrak{k}$ and $\mathfrak{g}$ be their Lie algebras. Take a suitable Cartan-Weyl basis $\left\{X_{\alpha}\right\}_{\alpha \in \Delta} \cup\left\{H_{i}\right\}_{i=1}^{r}$ for the root system which determines a Cartan involution with $\mathfrak{k}$ as its real form. Let $\mathfrak{h}$ be the real span of $H_{i}$ 's. Belavin and Drinfeld [B-D, So] proved that all multiplicative Poisson structures [D, Lu-We] on $G$ are classified by elements of the form $a \boldsymbol{r}+\boldsymbol{v}$ with $a \in \mathbb{R}, \boldsymbol{v} \in \mathfrak{h} \wedge \mathfrak{h}$, and

$$
\boldsymbol{r}=(i / 2) \sum_{\alpha \in \Delta_{+}}\left(X_{-\alpha} \otimes X_{\alpha}-X_{\alpha} \otimes X_{-\alpha}\right) \in \mathfrak{g} \wedge \mathfrak{g} .
$$

For each fixed multiplicative Poisson structure on $G$, one can construct a corresponding family of quantum groups $K_{q}, q>1$, or more precisely their algebras $C\left(K_{q}\right)^{\infty}$ of regular functions. Here in this paper, we only work on the standard $K_{q}$ arising from the multiplicative Poisson structure with $\boldsymbol{v}=0$ and $a$ normalized to 1. We remark that some interesting results for the opposite cases with $a=0$ and $\boldsymbol{v} \neq 0$ [Le-So] have been obtained in [Ri3].

Recall that the ${ }^{*}$-algebra $C\left(S U(2)_{q}\right)^{\infty}$ is generated by $u_{i j}, 1 \leq i, j \leq 2$, satisfying $u^{*} u=u u^{*}=1, u_{22}=u_{11}^{*}, u_{12}=-q^{-1} u_{21}^{*}$ and there is an important representation $\pi_{0}$ of $C\left(S U(2)_{q}\right)^{\infty}$ on $\ell^{2}\left(\mathbb{Z}_{\geq}\right)$given by

$$
\left(\pi_{0}\left(u_{i j}\right)\right)_{i, j=1}^{2}=\left(\begin{array}{cc}
\alpha & -q^{-1} \gamma \\
\gamma & \alpha^{*}
\end{array}\right)
$$

with $\alpha\left(e_{j}\right)=\left(1-q^{-2 j}\right)^{1 / 2} e_{j-1}$ and $\gamma\left(e_{j}\right)=q^{-j} e_{j}$ for $j \geq 0$ [V-So, Wo1].

Let $\alpha_{i}, 1 \leq i \leq r$, be the simple roots given by the Cartan-Weyl basis. Then each $\alpha_{i}$ determines a canonical embedding $\phi_{i_{*}}: S U(2) \rightarrow K$ which induces a Hopf *-algebra epimorphism $\phi_{i}: C\left(K_{q}\right)^{\infty} \rightarrow C\left(S U(2)_{q}\right)^{\infty}$, and the Weyl group of $K$ is generated by reflections $s_{i}$ associated with the roots $\alpha_{i}$. Soibelman found that one-dimensional irreducible ${ }^{*}$-representations $\tau_{t}$ of $C\left(K_{q}\right)^{\infty}$ are parametrized by elements $t$ in the maximal torus $\mathbb{T}^{r} \subseteq K$ and all irreducible ${ }^{*}$-representations are given by $\left(\tau_{t} \otimes \pi_{i_{1}} \otimes \pi_{i_{2}} \otimes \ldots \otimes \pi_{i_{m}}\right) \Delta^{m}$ where $\pi_{i}=\pi_{0} \circ \phi_{i}, s_{i_{1}} s_{i_{2}} \ldots s_{i_{m}}$ is a reduced expression in the Weyl group, and $\Delta$ is the comultiplication on $C\left(K_{q}\right)^{\infty}$. On the other hand, $t S_{i_{1}} S_{i_{2}} \ldots S_{i_{m}}$ also classifies all symplectic leaves in the Poisson Lie group $K$, where $S_{i}=\phi_{i_{*}}(S)$ with

$$
S=\left\{\left(\begin{array}{cc}
\alpha & \gamma \\
-\gamma & \bar{\alpha}
\end{array}\right): \alpha \in \mathbb{C},|\alpha|<1, \gamma=\sqrt{1-|\alpha|^{2}}\right\}
$$

the prominent 2-dimensional symplectic leaf in $S U(2)$. Note that by this result, one can complete $C\left(K_{q}\right)^{\infty}$ to get a $\mathrm{C}^{*}$-algebra $C\left(K_{q}\right)$, and that there is a one-to-one correspondence between symplectic leaves $L=t S_{i_{1}} S_{i_{2}} \ldots S_{i_{m}}$ in $K$ and irreducible *-representations $\pi_{L}=\left(\tau_{t} \otimes \pi_{i_{1}} \otimes \pi_{i_{2}} \otimes \ldots \otimes \pi_{i_{m}}\right) \Delta^{m}$ of $C\left(K_{q}\right)^{\infty}$ (or of $C\left(K_{q}\right)$ ).

In the spirit of non-commutative geometry [C], we shall call the quotient algebra $C\left(L_{q}\right):=C\left(K_{q}\right) / \operatorname{ker}\left(\pi_{L}\right)$ the $\mathrm{C}^{*}$-algebra of functions on a quantum symplectic leaf $L_{q}$ of the quantum Poisson group $K_{q}$ (cf. [J-St]). We remark here that in some sense, $C\left(L_{q}\right)$ quantizes regular functions of $L$ which are not necessarily vanishing at the infinity of $L$ (cf. [Sh1]), and hence $L_{q}$ quantizes the closure of $L$. Although we may take a suitable ideal of $C\left(L_{q}\right)$ (eliminated by some irreducible representations of $C\left(K_{q}\right)$ ) to define a quantum (open) leaf, it will not change our result and so the above definition of quantum leaf is fine for our purpose. In fact, roughly speaking, since the closure of a symplectic leaf $L$ is a disjoint union of symplectic leaves, the 
concept of 'preserving the closures of symplectic leaves' is equivalent to 'preserving symplectic leaves'.

\section{Quantization of $K$ By $K_{q}$}

By a quantization of $K$ by $K_{q}$, we mean any unital linear map from $C(K)^{\infty}$ to $C\left(K_{q}\right), q>1$, where $C(K)^{\infty}$ is the ordinary algebra of regular functions on the real Lie group $K$. We say that a quantization of $K$ by $K_{q}$ is group-preserving if it is a coalgebra homomorphism, since the group structure of $K$ corresponds to the coalgebra structure on $C(K)^{\infty}$.

On the other hand, it is natural to call a quantization $\phi: C(K)^{\infty} \rightarrow C\left(K_{q}\right)$ leaf-preserving, if $\phi$ induces a quantization $\phi_{L}$ on each leaf $L$ so that the diagram

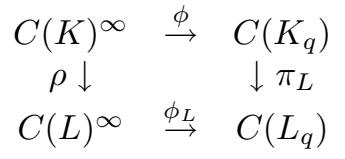

commutes, where $\rho$ is the restriction map to $L$ and $C(L)^{\infty}=\left\{\left.f\right|_{L}: f \in C(K)^{\infty}\right\}$. (Note that $f \in C(L)^{\infty}$ need not vanish at the infinity of $L$.) This definition is equivalent to the requirement that if $\left.f\right|_{L}=0$, then $\pi_{L}(\phi(f))=0$, for any $f \in C(K)^{\infty}$ and any symplectic leaf $L$ of $K$.

The above definition was actually motivated by the study of $\mathrm{C}^{*}$-algebraic deformation quantization of Poisson $S U(2)$. It was found that by quantizing functions on individual leaves $L$ of $S U(2)$ by classical Weyl quantization, one can get $\mathrm{C}^{*}$ algebraic deformation quantization $W_{h}$ of $S U(2)$ by $S U(2)_{q}$ which is automatically leaf-preserving [Sh1]. However it is hard to check directly if $W_{h}$ actually preserves the coalgebra structure. On the other hand, there are also examples of $\mathrm{C}^{*}$-algebraic deformation quantization which are group-preserving but not leaf-preserving [Sh2].

It is natural to ask if there are quantizations of $K$ by $K_{q}$ which are both grouppreserving and leaf-preserving so as to make an even closer connection between the geometric and the algebraic aspects of the theory of quantum groups. For the case of $K=S U(2)$, the problem was solved with a negative answer [Sh3]. In the following, based on that result, we shall give a negative answer for general cases.

\section{THE NON-EXISTENCE THEOREM}

In this section we prove the non-existence theorem and we refer readers to [So, Le-So] for further notation and results not reviewed in previous sections.

Before we prove the non-existence theorem, we need to make a remark about onedimensional irreducible ${ }^{*}$-representations $\tau_{t}$ found in [So]. Since the presentation in these papers is mainly aimed at the more complicated case of infinite-dimensional irreducible *-representations (but this point was not made clear in their context), one-dimensional representations $\tau_{t}$ were not explicitly treated. Actually a statement there about general irreducible ${ }^{*}$-representations is not correct when applied to the one-dimensional ones, which causes some confusion when one tries to work out more details about $\tau_{t}$. More precisely, it was stated [So] that for any irreducible *-representation $\pi$ (corresponding to a Schubert cell $X_{w}$ with $w$ in the Weyl group), the spectrum $\sigma\left(\pi\left(C_{-w \Lambda, \Lambda}^{\Lambda}\right)\right)$ of the operator $\pi\left(C_{-w \Lambda, \Lambda}^{\Lambda}\right)$ is of the form $E \cup\{0\}$ with $E \subset \mathbb{C} \backslash\{0\}$ a bounded set with no limit point in $\mathbb{C} \backslash\{0\}$. It has been verified that $\pi\left(C_{-w \Lambda, \Lambda}^{\Lambda}\right)$ is injective and normal. But when $w$ is the identity element $e$ of the Weyl group, $X_{w}$ is a singleton and $\pi$ is a one-dimensional irreducible ${ }^{*}$-representation. 
So $\sigma\left(\pi\left(C_{-w \Lambda, \Lambda}^{\Lambda}\right)\right)$ is a singleton different from $\{0\}$, which contradicts the above statement. In fact, the commutation relation used to conclude $0 \in \sigma\left(\pi\left(C_{-w \Lambda, \Lambda}^{\Lambda}\right)\right)$ in the above statement, namely,

$$
C_{-\mu, p, \Lambda}^{\Lambda} \cdot C_{-\lambda, i, \Lambda}^{\Lambda}=q^{-(\Lambda, \Lambda)+(\lambda, \mu)} C_{-\lambda, i, \Lambda}^{\Lambda} \cdot C_{-\mu, p, \Lambda}^{\Lambda}
$$

modulo $J_{2}(\lambda, \Lambda)$ with $\lambda=w \Lambda$ and $\mu<\Lambda$ [So], yields no information when $w=e$, since in this case, $C_{-\mu, p, \Lambda}^{\Lambda} \in J_{2}(\Lambda, \Lambda)=J_{2}(\lambda, \Lambda)$ and $\pi\left(C_{-\mu, p, \Lambda}^{\Lambda}\right)=0$.

Since one-dimensional representations $\tau_{t}$ were not explicitly constructed and analyzed in [So, Le-So], we give a detailed description of them which fits our framework in proving the main theorem, although this description comes from Levendorskii and Soibelman's work naturally and must be well known to experts like them.

First we give a constructive definition of $\tau_{t}$ which fits our need. Indeed, we can compose the Hopf ${ }^{*}$-algebra epimorphisms $\phi_{i}: C\left(K_{q}\right)^{\infty} \rightarrow C\left(S U(2)_{q}\right)^{\infty}$ and the one-dimensional irreducible *-representations $\tau_{\text {diag }\left(d, d^{-1}\right)}$ of $C\left(S U(2)_{q}\right)^{\infty}$ corresponding to the 0 -dimensional leaves $\left\{\operatorname{diag}\left(d, d^{-1}\right)\right\}$ of $S U(2), d \in \mathbb{T}$, to construct one-dimensional irreducible ${ }^{*}$-representations of $C\left(K_{q}\right)^{\infty}$. Here $\tau_{\operatorname{diag}\left(d, d^{-1}\right)}(u)=$ $\operatorname{diag}\left(d, d^{-1}\right)$ for $u=\left(u_{i j}\right)_{i, j=1}^{2}$, which satisfies $\tau_{\operatorname{diag}\left(d, d^{-1}\right)}\left(C_{-\omega, \omega}^{\omega}\right)=d$ for the standard fundamental weight $\omega$ of $\mathrm{su}(2)$. Thus $\left(\tau_{\operatorname{diag}\left(d, d^{-1}\right)} \circ \phi_{i}\right)\left(C_{-\omega_{j}, \omega_{j}}^{\omega_{j}}\right)$ equals $d$ if $i=j$ and equals 1 if $i \neq j$, where $\omega_{i}$ are the fundamental weights determined by the simple roots $\alpha_{i}$. On the other hand, for any $\Lambda=\sum_{i=1}^{r} m_{i} \omega_{i}$, we have

$$
\prod_{i=1}^{r} \pi\left(C_{-\omega_{i}, \omega_{i}}^{\omega_{i}}\right)^{m_{i}}=\pi\left(C_{-\Lambda, \Lambda}^{\Lambda}\right)
$$

for one-dimensional irreducible *-representations $\pi$ of $C\left(K_{q}\right)^{\infty}$ (with $w=e$ ) [So], which shows that $\pi$ is determined by the values $\pi\left(C_{-\omega_{j}, \omega_{j}}^{\omega_{j}}\right) \in \mathbb{T}$. So we may define, for each element $t$ in the maximal torus $\mathbb{T}^{r}$ of $K$, a one-dimensional irreducible *-representation by

$$
\tau_{t}=\left(\bigotimes_{i=1}^{r} \tau_{\operatorname{diag}\left(t_{i}, t_{i}^{-1}\right)} \circ \phi_{i}\right) \circ \Delta^{r-1}
$$

We claim that, for each $j$,

$$
\tau_{t}\left(C_{-\omega_{j}, \omega_{j}}^{\omega_{j}}\right)=t_{j}
$$

In fact, $\Delta^{r-1}\left(C_{-\omega_{j}, \omega_{j}}^{\omega_{j}}\right)$ is a sum of elementary tensors of the form

$$
\bigotimes_{i=1}^{r} C_{-\mu_{i-1}, p_{i-1}, \mu_{i}, p_{i}}^{\omega_{j}}
$$

with $\mu_{i} \in P\left(\omega_{j}\right), 1 \leq p_{i} \leq \operatorname{dim}\left(L\left(\omega_{j}\right)\right)_{\mu_{i}}$, and $\mu_{0}=\mu_{r}=\omega_{j}$. Since $\tau_{\operatorname{diag}\left(t_{i}, t_{i}^{-1}\right)} \circ \phi_{i}$ is a one-dimensional irreducible ${ }^{*}$-representation of $C\left(K_{q}\right)^{\infty}$, it eliminates $C_{-\mu, p, \omega_{j}, 1}^{\omega_{j}}$ with $\mu \in P\left(\omega_{j}\right) \backslash\left\{\omega_{j}\right\}$. Thus we get

$$
\tau_{t}\left(C_{-\omega_{j}, \omega_{j}}^{\omega_{j}}\right)=\prod_{i=1}^{r}\left(\tau_{\operatorname{diag}\left(t_{i}, t_{i}^{-1}\right)} \circ \phi_{i}\right)\left(C_{-\omega_{j}, 1, \omega_{j}, 1}^{\omega_{j}}\right)=t_{j},
$$

and so $\tau_{t}$ are all distinct and exhaust all possible one-dimensional irreducible *_ representations of $C\left(K_{q}\right)^{\infty}$. We note that $\tau_{\operatorname{diag}\left(d, d^{-1}\right)} \circ \phi_{i}=\tau_{(1, \ldots, 1, d, 1, \ldots, 1)}$, where $(1, \ldots, 1, d, 1, \ldots, 1)$ has $d$ in the $i$-th component. 
Finally we mention that when $K \subseteq G$ is of types $A, B, C$, or $D$, the algebra $C\left(K_{q}\right)^{\infty}$ is generated by entries of some $u=\left(u_{i j}\right)_{i, j=1}^{n}$ satisfying certain commutation relations and $\tau_{t}$ can be defined explicitly by $\tau_{t}(u)=t$ for $t$ in the maximal torus of $K[\mathrm{~J}-\mathrm{St}]$.

Theorem 1. There is no quantization of the Poisson group $K$ by $K_{q}$ which is both group-preserving and leaf-preserving.

Proof. We prove by contradiction. So assume that $\phi: C(K)^{\infty} \rightarrow C\left(K_{q}\right)$ is a quantization which is both group-preserving and leaf-preserving.

First we recall that the symplectic leaves of $S U(2)$ are either singletons $\ell=$ $\left\{\operatorname{diag}\left(d, d^{-1}\right)\right\}$ in the maximal torus of $S U(2)$ or of the form $L=\operatorname{diag}\left(d, d^{-1}\right) \cdot S$, where $d \in \mathbb{T}$. The embedding $\phi_{1 *}$ sends $\left\{\operatorname{diag}\left(d, d^{-1}\right)\right\}$ to $\{(d, 1, \ldots, 1)\}$ in the maximal torus $\mathbb{T}^{r}$ of $K$, and sends $\operatorname{diag}\left(d, d^{-1}\right) \cdot S$ to $(d, 1, \ldots, 1) \cdot \phi_{1_{*}}(S)=(d, 1, \ldots, 1)$. $S_{1}$. So according to Soibelman's classification of symplectic leaves of $K, \phi_{1_{*}}$ sends each symplectic leaf of $S U(2)$ onto a symplectic leaf of $K$. Furthermore, from the above discussion, we have

$$
\pi_{\phi_{1 *}(\ell)}=\tau_{(d, 1, \ldots, 1)}=\tau_{\operatorname{diag}\left(d, d^{-1}\right)} \circ \phi_{1}=\pi_{\ell} \circ \phi_{1}
$$

and

$$
\begin{gathered}
\pi_{\phi_{1 *}(L)}=\left(\tau_{(d, 1, \ldots, 1)} \otimes \pi_{1}\right) \circ \Delta \\
=\left(\tau_{\operatorname{diag}\left(d, d^{-1}\right)} \otimes \pi_{0}\right)\left(\phi_{1} \otimes \phi_{1}\right) \circ \Delta=\left(\tau_{\operatorname{diag}\left(d, d^{-1}\right)} \otimes \pi_{0}\right) \circ \Delta \circ \phi_{1}=\pi_{L} \circ \phi_{1}
\end{gathered}
$$

where the last $\Delta$ is the comultiplication on $C\left(S U(2)_{q}\right)$. Thus we get for any symplectic leaf $L$ of $S U(2), \pi_{\phi_{1_{*}}(L)}=\pi_{L} \circ \phi_{1}$.

Because of the above observation and the assumption that $\phi$ preserves leaves, we get a (unital) quantization $\psi$ of $S U(2)$ induced by $\phi$ shown in the following commuting diagram

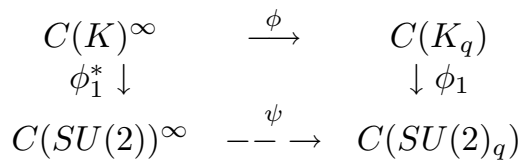

where $\phi_{1}^{*}(f)=f \circ \phi_{1 *}$ is the restriction of $f$ to the embedded $S U(2)$. In fact, if $\phi_{1}^{*}(f)=0$ or equivalently $\left.f\right|_{\phi_{1_{*}}(S U(2))}=0$, then for any symplectic leaf $L$ of $S U(2)$, we have $\left.f\right|_{\phi_{1_{*}}(L)}=0$, which implies $\pi_{\phi_{1_{*}}(L)}(\phi(f))=0$ and hence $\pi_{L}\left(\phi_{1}(\phi(f))\right)=0$. Thus we get $\phi_{1}(\phi(f))=0$ since $\pi_{L}$ classifies all irreducible ${ }^{*}$-representations of $C\left(S U(2)_{q}\right)$, and hence

$$
\psi\left(\phi_{1}^{*}(f)\right):=\phi_{1}(\phi(f))
$$

well defines a unital linear map from $C(S U(2))^{\infty}$ into $C\left(S U(2)_{q}\right)$.

Let $g=\phi_{1}^{*}(f) \in C(S U(2))^{\infty}$. If $\left.g\right|_{L}=0$, then $\left.f\right|_{\phi_{1_{*}}(L)}=0$, which implies $\pi_{\phi_{1_{*}}(L)}(\phi(f))=0$ and hence $\pi_{L}(\psi(g))=\pi_{L}\left(\phi_{1}(\phi(f))\right)=0$. Thus $\psi$ is also leafpreserving.

On the other hand, since $\phi, \phi_{1}$, and $\phi_{1}^{*}$ are coalgebra homomorphisms and $\phi_{1}^{*}$ is a surjection, it is not hard to see that the induced map $\psi$ is also a coalgebra homomorphism.

So in this way, we get a quantization of $S U(2)$ by $S U(2)_{q}$ which is both leafpreserving and group-preserving. But the non-existence of such a quantization for $S U(2)$ has been proved in [Sh3], and hence we get a contradiction. 


\section{REFERENCES}

[B-D] A. Belavin and V. Drinfeld, Solutions of the classical Yang-Baxter equation for simple Lie algebras, Functional Anal. Appl. 16 (1982). MR 84e:81034

[C] A. Connes, A survey of foliation and operator algebras, Proc. Sympos. Pure Math., vol. 38, Part I, Amer. Math. Soc., Providence, RI, 1982, pp. 521-628. MR 84m:58140

[D] V. G. Drinfeld, Quantum groups, Proc. I.C.M. Berkeley 1986, vol. 1, Amer. Math. Soc., Providence, RI, 1987, pp. 789-820. MR 89f:17017

[J-St] B. Jurčo and P. Šťoviček, Quantum dressing orbits on compact groups, Comm. Math. Phys. 152 (1993), 97-126. MR 94b:58008

[Le-So] S. Levendorskii and Y. Soibelman, Algebras of functions on compact quantum groups, Schubert cells and quantum tori, Comm. Math. Phys. 139 (1991), 141-170. MR 92h:58020

[Lu-We] J. H. Lu and A. Weinstein, Poisson Lie groups, dressing transformations and Bruhat decompositions, J. Differential Geom. 31 (1990), 501-526. MR 91c:22012

[Re-T-F] N. Yu. Reshetikhin, L. A. Takhtadzhyan, and L. D. Faddeev, Quantization of Lie groups and Lie algebras, Leningrad Math. J. 1 (1990), 193-225. MR 91d:17017

[Ri1] M. A. Rieffel, Deformation quantization and operator algebras, Proc. Sympos. Pure Math., vol. 51, Amer. Math. Soc., Providence, RI 1990, pp. 411-423. MR 91h:46120

[Ri2] Quantization and $C^{*}$-algebras, $C^{*}$-Algebras: 1943-1993, Contemp. Math., vol. 167, Amer. Math. Soc., Providence, RI, 1994, pp. 66-97. CMP 94:17

[Ri3] Compact quantum groups associated with toral subgroups, Contemp. Mathematics, vol. 145, Amer. Math. Soc., Providence, RI, 1993, pp. 465-491. MR 94i:22022

[Sh1] A. J. L. Sheu, Quantization of the Poisson SU(2) and its Poisson homogeneous space - the 2-sphere, Comm. Math. Phys. 135 (1991), 217-232. MR 91m:58011

[Sh2] _ Weyl quantization of Poisson SU(2), Pacific J. Math. (to appear).

[Sh3] _ Leaf-preserving quantizations of Poisson SU(2) are not coalgebra homomorphisms, Comm. Math. Phys. (to appear).

[Sh4] , Compact quantum groups and groupoid $C^{*}$-algebras, preprint.

[So] Ya. S. Soibelman, Algebra of functions on compact quantum group and its representations, Leningrad Math. J. 2 (1991), 161-178. MR 91i:58053a

[So-V] Ya. S. Soibelman and L. L. Vaksman, On some problems in the theory of quantum groups, Adv. Soviet Math., vol. 9, Amer. Math. Soc., Providence, RI, 1992, pp. 3-54. MR 93h: 17043

[V-So] L. L. Vaksman and Ya. S. Soibelman, Algebra of functions on the quantum group SU(2), Functional Anal. Appl. 22 (1988), 170-181. MR 90f:17019

[We] A. Weinstein, The local structure of Poisson manifolds, J. Differential Geom. 18 (1983), 523-557. MR 86i:58059

[Wo1] S. L. Woronowicz, Twisted SU(2) group: an example of a non-commutative differential calculus, Publ. Res. Inst. Math. Sci. 23 (1987), 117-181. MR 88h:46130

[Wo2] Compact matrix pseudogroups, Comm. Math. Phys. 111 (1987), 613-665. MR 88m:46079

Department of Mathematics, University of Kansas, Lawrence, Kansas 66045

E-mail address: sheu@kuhub.cc.ukans.edu 